\title{
Preliminary Orbits from Velocities with the CCD-Echelle Spectrometer at Lick
}

\author{
DANIEL M. POPPER \\ Department of Astronomy, University of California, \\ Los Angeles, CA 90024, USA
}

The Hamilton CCD-echelle spectrometer at the coude focus of the 3-m Shane telescope of the Lick observatory has been described by Vogt (1987). Ilustrations of some binary star spectra obtained with this instrument are given by Popper \& Nelson (1990, B-type binaries with large magnitude differences) and by Popper (1992, G-type eclipsing binaries), where typical cross-correlation functions are also shown.

In this progress report, I give some initial preliminary orbital results. First is the bright early B binary, VV Ori, the orbits of which have been the subject of considerable controversy (Duerbeck 1975, Andersen 1976, Popper 1981). According to Chambliss \& Leung (1982) and to the line ratios, the secondary component contributes about $10 \%$ of the light of the system in the visual region. Multiple (2 to 4) exposures of VV Ori have been obtained with the Hamilton on 7 nights. Velocities of the primary are determined from 13 lines, primarily of $\mathrm{OII}$ and $\mathrm{HeI}$ between 440 and $590 \mathrm{~nm}$. The only lines found to be useful for the secondary are HeI 597.5 and 492.1. Analysis of the velocities is complicated by the poorly determined variation of the center-of-mass velocity, with a range of roughly $26 \mathrm{~km} \mathrm{~s}^{-1}$ and a period of roughly 120 days (e.g., Duerbeck 1975). Preliminary orbits based on this material lead to masses 11.1 and $4.7 \mathcal{M}_{\odot}$, to be compared to Duerbeck's values of 7.7 and 3.4. It is primarily the high sig$\mathrm{nal} /$ noise ratio, over 600 , that enables this study to be carried out satisfactorily.

My principal program at Lick consists of a survey of spectra of eclipsing binaries that could have one or both components of type $\mathrm{G}-\mathrm{K}$ in the mainsequence band, where our knowledge of stellar masses and other fundamental properties is weak. The primary source of potential systems of this type is the Finding List for Observers of Interacting Binaries (Wood et al. 1980), with the IAU Information Bulletins on Variable Stars also proving useful. About $15 \%$ of the systems examined appear promising for the purpose, and a few have enough observatins for very preliminary orbits. The observations cover the region 442 to $682 \mathrm{~nm}$ in 46 spectral orders. Analsis is by cross-correlation with a spectrum of a star of well-determined radial velocity in the spectral-type range late $F$ to early $K$. Discussion of the determination of velocities in this manner is to be found in a number of papers in the Proceedings of IAU Colloquium 88, Stellar Radial Velocities. The software employed in this work is that developed recently for the echelle format at NOAO within the IRAF program. I am indebted to Mike Fitzpatrick and Frank Valdes of NOAO for their help and to UCLA graduate students Brant Nelson and Cecilia Barnbaum for tutoring. 
The difference of velocity between a component of a binary system and a standard star is given by

$$
\Delta V=K(\Delta p-\delta p),
$$

where $\mathrm{K}$ is the scale in $\mathrm{km} \mathrm{s}^{-1} \mathrm{pxl}^{-1}$ (close to 2.5 in our case); $\Delta \mathrm{p}$ is the separation in pixels obtained from cross-correlating the two spectra; and $\delta p$ is the pixel difference between the two corresponding ThAr lamp spectra. All of the quantities in the equation are independent of spectral order, which runs from 129 to 84 in the spectral region covered, provided the two observations are made during the same observing run. Because of the small spectral range, about $4.0 \mathrm{~nm}$, in a single order, the variation of $K$ along an order can be neglected. The only functions of the ThAr lamp spectra are to determine the value of $K$, which is quite stable over time, and of $\delta$ p. Conversion of the spectra to a log lambda scale is unnecessary and appears to degrade the precision of the results slightly. Tests cross-correlating the spectrum of one standard velocity star against another give vales of $\Delta V$ in excellent agreement with the standard values. [Equations (3) and (4) in Popper (1992) are not readily applied in IRAF and, in any case, are unnecessary refinements.]

Preliminary orbits from a limited number of observations of three systems are given in Table 1. The results for VV Ori are included. CV Boo and HD 84207 may be added to the very short list of G-type binaries with a component well determined to have a mass less than one solar mass, the others being $\alpha \mathrm{CrB}, \mathrm{FL}$ Lyr, HS Aur, and possibly UV Leo.

The last column of Table 1 gives the internal mean error of a single velocity, derived from the inter-agreement among the 40-odd orders. The striking dependence on period is in the sense expected. The corresponding value for a velocity difference between standard stars is $0.1 \mathrm{~km} \mathrm{~s}^{-1}$. The average external residual of a velocity relative to the derived orbit averages 2.2 times as large as the internal values given in the Table.

TABLE 1. Preliminary orbital elements.

\begin{tabular}{|lrccccc|}
\hline Binary & Mag & $\begin{array}{c}\text { Primary } \\
\text { type }\end{array}$ & $\begin{array}{c}\text { Period } \\
\text { (days) }\end{array}$ & $\begin{array}{c}\text { Primary } \\
\text { Mass }\end{array}$ & $\begin{array}{c}\text { Secondary } \\
\text { Mass }\end{array}$ & $\begin{array}{c}\text { Error } \\
\left(\mathrm{km} \mathrm{s}^{-1}\right)\end{array}$ \\
\hline CV Boo & 10.2 & G2: & 0.85 & 1.00 & 0.94 & 2.2 \\
HD 84207 & 9.5 & G2: & 1.37 & 0.96 & 0.94 & 1.2 \\
AD Boo & 9.8 & F9 & 2.07 & 1.37 & 1.17 & 0.7 \\
VV Ori & 5.3 & B1V & 1.49 & 11.1 & 4.7 & - \\
\hline
\end{tabular}
dation.

This investigation is supported by a grant from the National Science Foun- 


\section{REFERENCES}

Andersen, J. 1976, $A \& A, 47,467$

Chambliss, C.R. \& Leung, K.C. 1982, ApJS, 49, 531

Duerbeck, H.W. 1975, Astr. Ap. Suppl. 22, 19

Popper, D.M. 1981, ApJS, 47, 339

Popper, D.M. 1992, in Proc. Int. Colloq. on New Frontiers in Binary Star Research, ed. K.C. Leung, (San Francisco: Publ. A. S. P.), in press

Popper, D. M. \& Nelson, B. 1990, in CCD's in Astronomy. II, ed. A.G.D. Philip, D.S.

Hayes, and S. Adelman, (Schenectady: L. Davis), p. 26

Vogt, S.S. 1987, PASP, 99, 1214

Wood, F.B., Oliver, J.P., Florkowski, D.R., \& Koch, R.H. 1980, Publ. Dept. Astron. U. Florida, Vol. I 